\title{
Ausbildungsprofile erweitern
}

\section{Neues Berufsbild zwischen Arzt und Pflegekraft}

Im medizinischen Bereich sind in den vergangenen Jahren einige neue Berufsbilder entstanden - auch auf akademischem Niveau. Eines davon ist der „Physician Assistant“, der Arztmitarbeiter - wörtlich übersetzt. Doch das erklärt den neuen Beruf, der noch keinen deutschen Begriff hat, nicht ausreichend. Der „Physician Assistant“ erwirbt in einem dreijährigen BachelorStudium medizinische, aber auch organisatorische Kenntnisse und nimmt eine Zwischenstellung zwischen Arzt und Pflegekraft ein. Deutschlandweit gibt es bisher eine Handvoll Ausbildungsmöglichkeiten für den Beruf, einige private Hochschulen bieten den Ausbildungsgang an, eine staatliche Hochschule in Baden-Württemberg, und ab dem Wintersemester wird es auch einen dualen Studiengang an der Staatlichen Studienakademie in Plauen (Sachsen) geben. Ziel ist es nach Angaben der Studienakademie, eine „akademische Ausbildung auf einem Niveau zwischen den Qualifizierungsstufen Arzt und Pflegepersonal für einen Einsatz in Kliniken“ anzubieten. Eigenverantwortlich dürfen die „Phycisian Assistants“ nicht arbeiten, sondern nur delegierbare ärztliche Tätigkeiten ausführen. Entlastung sollen sie vor allen Dingen bei bürokratischen Arbeiten bringen, um für Ärzte Freiräume zur Behandlung von Patienten zu schaffen. In dem dreijährigen Modellvorhaben wird das Studienangebot aktiv durch die Landesärztekammer begleitet und evaluiert.

sas

\section{Gesundheits-IT}

\section{Lieferverzögerungen bringen Gröhes Zeitplan ins Wanken}

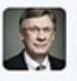

troppe

@BMG_Bund Verträge der@gematik_de

mit Industrie müssen alle einhalten.

Digitalisierung d. Gesundheitswesens

muss fristgerecht kommen.

Gesprach zeigen

Stroppe clutzStroppe - 27 iul

Start Gesundheits-IT wurde bis Juni nicht in Frage gestellt. Jetzt keine Ausreden. Probebetrieb soll dieses Jahr beginnen. BMG Bund

Staatssekretär Lutz Stroppe via Twitter

Ende Mai hat das Bundeskabinett den bei Ärzten und Zahnärzten umstrittenen Gesetzentwurf für sichere digitale Kommunikation und Anwendungen im Gesundheitswesen (kurz: EHealth-Gesetz) beschlossen. Darin enthalten: ein strenger Zeitplan für die Einführung der digitalen Infrastruktur. Doch dieser Zeitplan ist offenbar ins Wanken geraten.

In einem Pressestatement stellte Bundesgesundheitsminister Hermann Gröhe noch fest: "Viel zu lang wurde schon gestritten. Jetzt gehört endlich der konkrete Nutzen der elektronischen Gesundheitskarte für den Patienten in den Mittelpunkt. Deshalb machen wir Tempo durch klare gesetzliche Vorgaben, Fristen und Anreize, aber auch Sanktionen, wenn blockiert wird."

Acht Wochen später scheint dieses Versprechen schon wieder Makulatur zu sein: Denn Informationen der Gesellschaft für Telematikanwendungen der Gesundheitskarte (gematik) zufolge verzögert sich der für November geplante Start der abschließenden Tests in zwei Versuchsregionen wegen Lieferschwierigkeiten der Industrie. Der Online-Test könne „frühestens im ersten Quar- tal 2016" erfolgen, zitiert die Frankfurter Allgemeine Zeitung (FAZ) Ende Juli aus einem der Redaktion vorliegenden Schreiben der gematik an Gesundheitsstaatssekretär Lutz Stroppe.

\section{Konnektoren müssen nachgerüstet werden}

Stein des Anstoßes sind die sogenannten Konnektoren, welche die Karten lesen und die Arzt- und Zahnarztpraxen sicher mit dem Netz verbinden. Bis Anfang Juli sollte die Industrie Musterexemplare beim Bundesamt für die Sicherheit in der Informationstechnik (BSI) zur Prüfung einreichen. Rund 1000 Praxen sollten anschließend für den großen Systemtest ausgerüstet werden. Das funktioniere nun nicht, denn keiner der fünf Hersteller habe die Geräte fristgerecht geliefert, berichtet die FAZ. Nun streiten die Beteiligten, wer für die Panne verantwortlich ist. Die gematik bezichtigt die Industrie, die wiederum sieht das BSI in der Verantwortung. Erst vor Kurzem habe das BSI neue Sicherheitsanforderungen gestellt, die die Hersteller umsetzen müssten, führt der Bundesverband Gesundheits-IT (BVItG) an.

\section{Politik hält am Zeitplan fest}

Die Politik will von all dem nichts wissen und hält offiziell an ihrem Zeitplan fest. Über den Nachrichtendienst Twitter lässt Staatssekretär Stroppe wissen: Die "Digitalisierung des Gesundheitswesens muss fristgerecht kommen. Jetzt keine Ausreden." Findet die Erprobung nicht statt, geraten auch alle anderen Fristen ins Rutschen. Für Gröhe wäre das ein Desaster. Die Krankenkassen haben bisher Versichertengelder in Höhe von rund einer Milliarde Euro allein in die elektronische Gesundheitskarte investiert. Sie gilt als Schlüssel für den Zugang zur geplanten Datenautobahn im Gesundheitswesen. Ihr bisher einziger erkennbarer Mehrwert: ein Foto des Versicherten. Antje Schmilgus

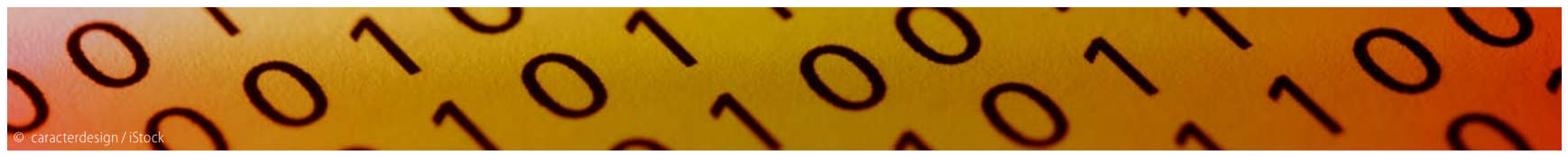

